\title{
Social Engineering: I-E based Model of Human Weakness for Attack and Defense Investigations
}

\author{
Wenjun Fan ${ }^{1+}$, Kevin Lwakatare ${ }^{2}$ and Rong Rong ${ }^{3}$ \\ ${ }^{1+}$ Department of Telematics Engineering, ETSI Telecommunication Technical University of Madrid, Madrid, Spain \\ ${ }^{2}$ Department of Computer Science, TUT Centre for Digital Forensics and Cyber Security Tallinn University of \\ Technology, Tallinn, Estonia \\ ${ }^{3}$ IE Business School, Madrid, Spain \\ E-mail: efan@dit.upm.es ${ }^{1+}$, kevinlwaks@gmail.com² ${ }^{2}$,rong.mm2013@alumni.ie.edu ${ }^{3}$
}

\begin{abstract}
Social engineering is the attack aimed to manipulate dupe to divulge sensitive information or take actions to help the adversary bypass the secure perimeter in front of the information-related resources so that the attacking goals can be completed. Though there are a number of security tools, such as firewalls and intrusion detection systems which are used to protect machines from being attacked, widely accepted mechanism to prevent dupe from fraud is lacking. However, the human element is often the weakest link of an information security chain, especially, in a human-centered environment. In this paper, we reveal that the human psychological weaknesses result in the main vulnerabilities that can be exploited by social engineering attacks. Also, we capture two essential levels, internal characteristics of human nature and external circumstance influences, to explore the root cause of the human weaknesses. We unveil that the internal characteristics of human nature can be converted into weaknesses by external circumstance influences. So, we propose the I-E based model of human weakness for social engineering investigation. Based on this model, we analyzed the vulnerabilities exploited by different techniques of social engineering, and also, we conclude several defense approaches to fix the human weaknesses. This work can help the security researchers to gain insights into social engineering from a different perspective, and in particular, enhance the current and future research on social engineering defense mechanisms.
\end{abstract}

Index Terms-Social Engineering, Semantic Attacks, Information Security, Data Privacy, Hacking Techniques, Human Weaknesses.

\section{INTRODUCTION}

Information security and privacy are very important to personal assets, corporate properties, and even state secrets, which across the globe are facing various hacking threats. In modern society, people use various digital equipments, such as cell phones, laptops, tablet pads and personal computers, connected by the Internet to communicate with each other and share information. Hence, modern information security becomes increasingly interconnected and dependent on IT security [1][2]. The IT security includes not only protecting the organization systems from being attacked but also preventing the system-related human or users from being tricked, in order to avoid leaking valuable information.

On one hand, due to the intelligence of blackhat community, there are many hacking techniques, such as buffer overflow, SQL injection and cross-site scripting (XSS), which can be used to attack the computer systems for accessing the sensitive information [3]. These attacks depend on exploiting the vulnerabilities of the software systems, which can be addressed by timely system update and supplementing the production system with security tools like firewall and intrusion detection system (IDS). On the other hand, some hackers pioneered the art of human hacking (also called phreakers in some earlier articles [4]) known as social engineering (SE) attacks to deceive the dupe in order to get valuable information, such as account names, ID numbers and even passwords, which can be further used to bypass the access control and evade intrusion detection. Hence, social engineering attacks focus on the human element's psychological vulnerabilities rather than the traditional technical ones.

The SE attacks are much more difficult for system administrators to defend against. At present, a large percentage of information security depends on the human rather than the technical security measures. According to the Verizon 2015 DBIR report [5], humans account for $90 \%$ of security incidents. A recent research report from Ponemon, sponsored by Wombat Security Technologies [6], also concludes that the average 10,000-employee company spends $\$ 3.7$ million a year dealing with phishing attacks. Symantec 2015 Internet Security Threat Report (ISTR) [7] also shows that five out of six large $(2,500+$ employees) companies are targeted by spearphishing attacks during 2014 - a 40-percent increase over the previous year; small and medium-sized businesses also see an uptick, with attacks increasing by 26 percents and 30 percents respectively. Thus, protection of sensitive information is vitally important to governments and organizations. Although the effectiveness of protecting information is increasing, human element is still susceptible to manipulation and is the weakest link.

Therefore, the objective of this paper is to make an effort to gain an insight into the social engineering 
research area. The contributions of this paper can be summarized as follows:

1) We capture two essential levels, the internal characteristics of human nature and the external circumstance influences, which shape human psychological states, and propose a novel I-E based model of human weakness.

2) We apply the I-E based model of human weakness to analyze the typical social engineering attack techniques in order to get insights into the social engineering attacks.

3) According to the model, we also suggest some social engineering defense measures to fix the human weaknesses for facilitating information security and privacy.

The remainder of this paper is organized as follows: section 2 reviews the related work; section 3 provides an overview of social engineering to identify the importance of human weakness; section 4 proposes a novel I-E based model of human weakness; section 5 analyzes the SE attack techniques in terms of the I-E based model; section 6 suggests some SE defense measures; section 7 makes a conclusion.

\section{RELATED WORKS}

\section{A. Social Engineering Taxonomies}

At present, there are plenty of materials [8][9], introducing social engineering attacks where security researchers can learn the concepts, the attack techniques, the interesting real cases, etc. Studying the dedicated taxonomies is another way to know well over a study field. To our knowledge, a decade ago, though there were a number of taxonomies of network attacks [10][11], few taxonomies were specially designed for social engineering attacks. Thereafter, some succeeding taxonomies of network attacks began to consider the classifications of social engineering. For example, Simmons et al. [12] proposed a taxonomy called AVOIDIT, which classified cyber attacks into six categories-attack vector, operational impact, defense, informational impact, and attack target. Attack vector is the vulnerability or path used to compromise a system, such as misconfiguration, buffer overflow, insufficient authentication validation etc. One of the subcategories of attack vector is social engineering. Another taxonomy [13] proposed by Van Heerden et al. consist of twelve classes, each containing multiple subclasses. Social engineering is one of the subclasses of the class "Attack Mechanism". Hence, both of AVOIDIT's and Van Heerden's taxonomies simply regarded social engineering as one of the attack methods but did not unveil the technique details about social engineering attacks.

In recent years, several novel taxonomies focused on social engineering attacks have been proposed, which can help us learn more details. In 2015, Krombholz et al. proposed a novel taxonomy [14] aimed to classify social engineering attacks. This taxonomy proposed three main categories for dissecting social engineering, and they are "channel, operator and type". The channel means the medium where the SE attacks conduct. It includes e-mail, instant message, telephone, VoIP, social network, cloud and website. The operator indicates the actor who launches the SE attacks, which can be human or software. The type refers to the approach that the SE attacks take. The taxonomy includes four approaches: physical, technical, social and socio-technical. Furthermore, the author summarized seven representative SE attack vectors (or scenarios): phishing, dumpster diving, shoulder surfing, reverse social engineering, waterholing, advanced persistent threat and baiting. Nevertheless, the author mentioned the fact that the each specific SE attack scenario had not been technically exhausted. In order to verify the taxonomy, the author applied it to these representative attack scenarios, which proves that the taxonomy works well in analyzing these typical SE attack vectors. Indeed, it is a scenario-driven taxonomy, which draws out the attack characteristics from the actual attack scenarios and then categories these characteristics into taxonomy. This taxonomy is designed mainly from the attack point of view, however, it lacks the main cause of social engineering attacks.

Another recent novel taxonomy of SE attacks was proposed by Heartfield and Loukas [15]. It adopts three distinct control stages-orchestration, exploitation and execution, as the basic categories of the taxonomy. For each stage, it poses questions that can help develop the technical protection mechanisms. The answers to these questions compose the corresponding categories, which consequently establish the whole taxonomy. The orchestration consists of target type (target of choice or opportunity), attack mode (manual or automated), and attack approach (software, hardware without software or hardware with software). The exploitation includes the deception vector (cosmetic, behavior or hybrid) and the manipulation interface (user interface or programmatic interface). The execution is comprised of execution steps (single or multiple) and attack persistence (one-off or continual). Furthermore, this taxonomy depicts several mutual-exclusive subcategories whose characteristics should be considered for developing the technical protection mechanisms. The taxonomy is not exhaustive and can be expanded based on the three main categories. Also, it is evaluated by being applied to 30 different attacks observed in the wild, which is aimed to help develop the technical protection mechanisms. However, the taxonomy adopts the definition of the three distinct control stages of orchestration, exploitation and execution as suggested by CESG [16], which aims to describe common cyber attacks instead of social engineering attacks. Hence, the categories of this taxonomy are more related to common cyber attacks than to social engineering attacks, which has some specific concerns that should be taken into account.

In addition, Mouton et al. [17] proposed an ontological model to define the social engineering domain and 
offered important insights into the various SE attack methods. The set of the categories provided by this social engineering ontology can be considered as a taxonomy as well. This work is also on the side of attacks to analyze social engineering. There is little consideration and analysis on the weaknesses from human element. Also, some categories concluded by the authors are very general for common cyber attacks. For example, the values of the class "target" and the class "goal" are also valid for the common cybercrimes.

\section{B. Social Engineering Conceptual Models}

A taxonomy is also a conceptual model. In this subsection, several dedicated conceptual models of social engineering will be presented.

In the book [4], Mitnick ever proposed a conceptual model from the perspective of attackers, to describe the social engineering attack cycle (SEAC). But the SEAC is explained too briefly and lacks many details. Based on that, Nohlberg and Kowalski [18] proposed a new model to describe the cycle of deception, which merges attacker, defender and victim. In the model each cycle has five steps. If an attacker is not able to meet the requirement of any step of the attacker cycle, his attack will fail. Similarly, if one of the steps in the defense cycle can stop the attacker, the attack will fail as well. Otherwise, the attacker will be successful and even is going to be able to do it again. This model can be used to build defenses or to map and describe an attack. Mouton et al. [19] proposed another social engineering attack framework combining their previously proposed SE ontological model [20] and extending Mitnick's social engineering attack cycles through specifying attack steps. It provides full details of every attack step and can map historical SE attacks into a standardized format.

A system archetype is also a good way to conceptualize the warfare framework of social engineering, through describing the relations between the system, the countermeasures and the intruder. Gonzalez et al. [21] uses system archetypes as the idealized patterns to describe the main modes of social engineering attacks. From each of the attack and the defense perspectives, the system archetypes presented unveil two feedback loops, called controlling balancing (B) loop and reinforcing (R) loop, whose four basic combinations can be used to describe the intended consequence (IC) of the social engineering attack and the unintended consequence (UC) of the organizational defense. The UC is the result of the organizational reaction to the SE attacks. However, SE attackers also have the solution loop (SOL) to deal with the organizational reaction, and always seek ways to outsmart the single-loop defense lines. So, the paper suggests designing organizational security controls which can provide multi-layer feedback against the combined action of SE attacker's IC and SOL.

The system archetype approach is good at conceptualizing the SE to a high level of abstraction. However, the power of its analysis remains questionable in terms of clarifying the techniques in detail. Tetri and Vuorinen [22] proposed a conceptualization of SE which consists of different dimensions of SE that can be used to exam the techniques of social engineering. Through reviewing the techniques used in actualizing the attacks, the paper extracts three different dimensions of SE techniques: persuasion, fabrication and data gathering. After that, it proposes an abstract SE framework, intruder-techniques-dupe. The authors emphasized that in real scenario the SE attacker would use multidimensional approaches to attack an organization, which proves that, in a particular case, the information security policy is the weakest link rather than the human element.

Besides, in particular, Abraham and Smith developed a framework [23] which showed that the steps social engineering malware executes can be successful. Indeed, this paper reveals some malware activated by social engineering channels, which include psychological and technical ploys. The psychological techniques include some persuasive tactics as well, such as using the victim's curiosity, empathy, excitement, fear and greed. The authors claimed that although it is important for organizations to build comprehensive information security program, the SE malware cannot be mitigated by organizations alone, instead, the shared responsibility of governments, ISPs, end users, and international bodies is needed to combat SE malware.

\section{AN OVERVIEW OF SOCIAL ENGINEERING ATTACK}

A common network intrusion can be divided into five steps - reconnaissance, scanning, exploiting, gaining access and maintaining access. For social engineering attacks, Kevin Mitnick's model [4] proposed another five steps - researching, developing trust, exploiting trust and utilizing information. The main difference between these two is that the human element is the weakest link in the SE attacks and is exploited by the attackers. Hence, we mainly focus on the differences between the SE attacks and the common cyber attacks, and present an overview of the SE attacks. The elements and workflow of the SE attacks are shown in Fig.1.

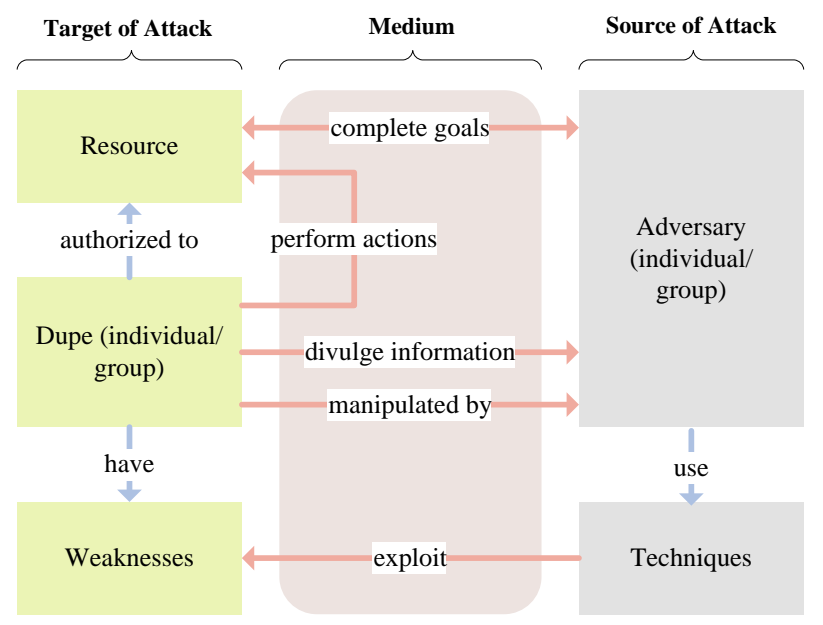

Fig.1. Elements and Workflow of the Social Engineering Attacks

A social engineering attack is aimed to manipulate 
human into performing actions or divulging confidential information so that the target resource can be accessed to complete a goal. The goal could be financial gains, unauthorized access and service disruption [17]. In order to achieve the goal, an adversary has to trick someone, who is authorized to access the resource, into providing sensitive information or breaking normal security procedures according to his manipulation. The source of attack is an individual or a group of adversaries who often use different social engineering techniques. The adversary known as social engineer is often entrenched by techniques of both IT technology and social psychology [14][15]. Those social engineering techniques often rely on some medium to attack the human weaknesses. The medium could be direct interactions such as face-to-face interview or communication over the telephone, or indirect interaction through letters, emails and websites, or even unidirectional interaction, e.g. leaving an USB on the ground to wait the dupe to pick it up [14][17]. Also, the dupe could be an individual or a group of victims, since SE threatens not only individuals but also companies, organizations, and governments. After a successful exploitation of the weaknesses, the dupe will be manipulated by the adversary. The dupe will divulge sensitive information for accessing the resource or even take place of the adversary to take malicious actions so that the adversary can complete the malicious goal. The whole process of doing that is known as social engineering attack.

Though security measures have made some fraudulent activities more difficult to conduct, the smart and skilled social engineers can excavate new opportunities to overcome them. Hence, the knowledge of both sides, i.e. attack and defense, is needed to do research about social engineering. From the workflow of SE attack, we can discover that the human weakness is a link of strategic importance to both the attack and the defense sides. In order to frustrate the exploitation, it is necessary to fix the human weakness. In contrast, social engineers need to exploit the weakness to complete the goal. Thus, it is reasonable to consider the human weakness as the foundation for investigating the SE attack and defense. In the next section, we will propose the dissection of the root cause of the human weakness in order to provide our insights into the social engineering.

\section{I-E BASED MODEL OF HUMAN WEAKNESS}

The topic of human weaknesses is a big subject, which includes not only psychology but also biology and even some principles related to sociology, economics etc. For example, Richard Dawkins's book "The Selfish Gene" [24] explains a lot of altruistic behaviors in the nature, especially the relationship between the relatives: an organism may take big risks to protect its relatives, and it does this because its relatives share similar genes so their safety is good for the genes' spreading. . This infers that a human often prefers to trust his families or relatives, but it does not guarantee that those people will never deceive the human. Another case is depicted in the Dale
Carnegie's best-selling book -"How to win friends and influence people" [25], which combines age-old truisms with the emerging field of psychology to give an instruction in handling people, winning friends, bringing people to your way of thinking, being a great leader and even navigating home life successfully. Carnegie presents the use of others' egotistical tendencies to one's advantage to get the success of building trust. Though this handbook is not used for social engineering, the way to develop trust between humans is the same.

Therefore, we can discover that the success of manipulating human is often achieved when some characteristics of human nature are triggered by some external influences and converted into weaknesses and then exploited by the SE techniques. So, based on this discovery, we capture two essential levels (or elements) shaping human psychological states - the internal characteristics of human nature and the external circumstance influences - which trigger the human weaknesses. We name it I-E based model of human weakness (Fig.2. graphically shows this model).

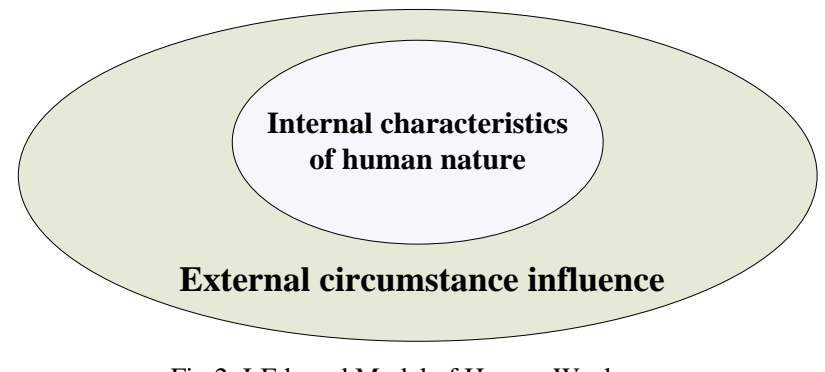

Fig.2. I-E based Model of Human Weakness

We will describe the internal characteristics of human nature and the external circumstance influences respectively in the next subsections.

\section{A. Internal Characteristics of Human Nature}

There are many types of characteristics of human nature. From the psychological point of view, they can be roughly divided into two big categories: positive and negative. Also, we cite the Seven Virtues and Sins in the Catholic catechism to make up the cardinal characteristics of human nature in both psychology categories respectively. The positive and negative psychological characteristics are described as follows:

1) Positive characteristics refer to the bright-side of personality traits.

- Chastity: discretion of sexual conduct according to one's state in life; the practice of courtly love; cleanliness by cultivated good health and hygiene, and maintained by refraining from intoxicants.

- Temperance: constant mindfulness of others and one's surroundings; practicing self-control, restraint, abstinence, moderation and deferred gratification.

- Charity: generosity and helpfulness especially toward the needy or suffering; aid given or voluntary giving of help to those in need; benevolent goodwill or love of humanity. 
- Diligence: a zealous and careful nature in one's actions; decisive work ethic, steadfastness in belief, fortitude and the capability of not giving up.

- Patience: building a sense of peaceful stability and harmony rather than conflict, hostility and antagonism; resolving issues and arguments respectfully, as opposed to resorting to anger and fighting.

- Kindness: compassion and friendship for its own sake; empathy and trust without prejudice or resentment; unselfish love and voluntary kindness without bias or spite; having positive outlooks and cheerful demeanor; to inspire kindness in others.

- Humility: a spirit of self-examination; a hermeneutic of suspicion toward yourself and charity toward people you disagree with; Modest behavior, selflessness, and the giving of respect; the courage of the heart necessary to undertake tasks that are difficult, tedious or unglamorous, and to graciously accept the sacrifices involved.

\section{2) Negative characteristics indicate the dark-side of} personality traits.

- Lust: it is usually thought of as intense or unbridled sexual desire, which leads to fornication, adultery, rape, bestiality and other immoral sexual acts.

- Gluttony: it is the overindulgence and overconsumption of anything to the point of waste.

- Greed: also known as avarice, cupidity or covetousness, is like lust and gluttony, a sin of desire. However, greed is applied to an artificial, rapacious desire and pursuit of material possessions.

- Sloth: it refers to a peculiar jumble of notions, dating from antiquity and including mental, spiritual, pathological and physical states. It may be defined as absence of interest or habitual disinclination to exertion.

- Wrath: it can be defined as uncontrolled feelings of anger, rage and even hatred, often revealing itself in the wish to seek vengeance. In its purest form, it presents with injury, violence, and hate which may provoke feuds that can go on for centuries.

- Envy: like greed and lust, it is characterized by an insatiable desire. It can be described as a sad or resentful covetousness towards the traits or possessions of someone else.

- Hubris: the negative version of pride is considered; it describes a personality quality of extreme or foolish pride or dangerous over-confidence; a feeling of deep pleasure or satisfaction derived from one's own achievements and the achievements of one's close associates, or from one's qualities or possessions that are widely admired.

We maintain that all other personality traits can be attributed to those fourteen characteristics. For example, curiosity is often originated from some desires, such as Lust, Greed and Envy; one's kind-heart is often based on the Kindness and the politeness relies on the Humility; the incaution arises from the mental Sloth. Also, we believe the fact that any common people have those fourteen characteristics of human nature. Usually, those characteristics are implicit, but under some circumstance influences, they will become increasingly explicit and convert into human weaknesses which can be used by the social engineer. In the next subsection we will focus on describing those external circumstance influences.

\section{B. External Circumstance Influences}

As stated, social engineering is a social exercise, and the attackers usually exploit the victims' weaknesses or psychological vulnerabilities, to get the attack success. An external circumstance influence is often an intensive impact from the environment where the dupe locates. The external circumstance influences can stimulate or trigger the psychological characteristics and convert them into human psychological weaknesses, which later can be considered as the targets of SE attack. So, in this subsection we describe the related external circumstance influences for social engineering attacks, and we summaries them as follows:

- Strong affect: it is an impact using a heightened emotion, such as feeling a strong sense of surprise, anticipation or even anger, as a powerful distraction of the victim's ability to evaluate and think logically when arguments are being presented. This can stimulate one's characteristics such as greedy, lust, gluttony, envy etc.

- Overloading: it refers that the victim has too much information to process, but does not have enough time to evaluate it. Hence, this is an influence to impair the victim's ability to process and scrutinize the arguments so that he or she is more willing to accept the arguments that should have been challenged. This can impact one's sloth, wrath, envy etc.

- Reciprocation: this influence indicates the social interaction rule, if someone gives us something or promises us something, we should return the favor. The reasoning follows that people are more willing to comply with a request if the requester has treated them favorably in the past. So, this can trigger one's charity, humility, kindness etc.

- Deceptive Relationships: this influence indicates that the attacker builds a fabricated relationship with the dupe in order to increase the chance that the dupe divulges private information to the attacker. The reason is that people are more willing to comply with requests from friends or someone they like and perform activities under a legitimated and trustworthy relationship. So, one way of doing this is sharing information through discussing about a common enemy. Another example is that the attacker appears as if he is very much like the target, e.g., they have the same interests or desire the same things out of life. This can influence almost all the cardinal characteristics.

- Diffusion of Responsibility and Moral Duty: this 
influence means victims are more willing to accept requests or perform actions when they feel that it is none of their business or they will not be held solely responsible for their actions. Hence, this can trigger the characteristics such as sloth, charity, humility, kindness, etc.

- Authority: it indicates that people will easily respond to the requests given by the people with more authority than themselves. This can influence one's humility, patience etc.

- Integrity and consistency: this influence refers that people have a tendency to follow the commitments and comply with the requests that are consistent with their thoughts, even though the commitments may not be very wise at the first place. This influence can trigger one's sloth, greed, lust, gluttony, envy etc.

- Social validation: this influence means that victims are easier to comply with the requests if they are regarded as the socially correct things to do. This can influence one's humility, charity, kindness etc.

- Scarcity: this influence presents that people are more likely to comply with a request that is scarce or decreasing in availability. The reason hiding behind is that people subconsciously approve the fact that objects are valued because of their rarity. So, this can impact one's greed, gluttony, lust, envy, diligence etc.

These external circumstance influences could objectively exist around the dupe or be subjectively constructed by the social engineer. If the dupe submerges into these scenarios, the probability of being exploited by the adversary will be very high. Additionally, there could be many other external circumstance influences and we can not enumerate all of them here. In the next section, we will analyze the SE attack techniques according to the I-E based model of human weakness.

\section{ATtACK TECHNIQUES}

\section{A. Descriptions of Techniques}

The SE attack techniques (also known as attack vectors in the paper [14]) represent the approaches used to exploit the human weaknesses. We classify them into four categories: physical, technical, social and hybrid.

1) Physical approaches refer to that the adversary performs some physical activities to gather information.

- Dumpster diving: it represents the action of digging through trash at corporations to search for sensitive data.

- Shoulder surfing: it indicates the observation techniques, such as looking over someone's shoulder, for the sake of getting security information.
2) Technical approaches refer to the technical actions mainly carried out over the Internet to gather sensitive information.

- Phishing: it is the attempt to acquire sensitive information, such as username, passwords, credit card details etc., or to make someone to act in a desired way by masquerading as a trustworthy entity in an electronic communication. The general phishing will attack a group of targets randomly. However, the spear-phishing focuses on attacking some specific individuals or cooperators, thus it requires the adversary to gather information on the intended targets beforehand. So, the spearphishing needs more efforts but also has a higher success rate than the general phishing attacks.

- Waterholing: it refers that the adversary compromises the websites which are often browsed or are likely to be of interest to the targets of choice, and infects the target victims with malware, and then waits for them getting infected at the waterhole.

- Baiting: it is like the real-world Trojan Horse that exploits the victims' greed and curiosity by the malware - infected temptation, which could be physical media or software and online items. The baiting attack is very similar to the phishing attack, while the baiting is more like a gift or a good which is left somewhere and can be found by the victims.

3) Social approaches rely on socio-psychology to manipulate the victims in order to get sensitive information.

- Persuasion: it is aimed to get a victim to comply with an inappropriate request making them perform some illicit actions out of some psychological weaknesses, such as purported authority. One representative persuasion is diversion theft, which is also known as "Corner Game" or "Round the Corner Game". It is a "Con" exercised by professional thieves, normally against a transport or courier company. The objective of diversion theft is to persuade the persons responsible for a legitimate delivery that the consignment is requested elsewhere - hence, "round the corner".

- Pretexting: it refers to the art of creating and using a fabricated scenario (the pretext) that can be used to increase the chance the dupe divulges information or performs actions which would otherwise be unlikely in ordinary circumstances. In comparison with the persuasion, pertexting stands for deceiving the dupe though using some of the techniques, such as impersonation, namedropping, using false ID etc.

- Quid pro quo: it means "something for something" or "this for that" in Latin, which refers that the SE attackers promise a benefit in 
exchange for information. This benefit usually assumes the form of a service, whereas baiting frequently takes the form of a good.

- Reverse social engineering: it is a type of attacks calling back when the victim needs the help from someone who claimed that he can solve the problem. It relies on the trust established between the attacker and the victim, which allows the attacker to gain the privileged information.

4) Hybrid approaches refer to the exploiting techniques consisting of multiple different single approaches described above.

- Tailgating: also known as "piggybacking", it refers to the type of attacks conducted by the adversary who lacks the proper authentication and seeks entry to a restricted area through following a person who has the legitimate access. These restricted areas, i.e. organizations and corporations, are often secured by unattended and electronic access control such as RFID-based entrance guard card. For instance, the adversary impersonates a delivery driver and waits outside a building. When an employee appears to gain the security's approval and opens the door, the adversary will hold the door open, or the employee may hold the door open for the trailing adversary following common courtesy, or the attacker may even ask the employee to hold the door open while the legitimate employee may fail to ask for identification for some reasons, such as a fabricated assertion that the attacker has forgotten or lost the appropriate identity token.

- Vishing: known as phone phishing, it is the act using the telephone in an attempt to scam the dupe into surrendering private information that will be used for identity theft. The scammer usually pretends to be a legitimate business, and fools the victim into thinking that he or she will profit.

\section{B. Analysis of techniques}

In this subsection, we analyze the SE attack techniques by applying the proposed I-E based human weaknesses. Table 1. shows the comparison between those SE attack techniques in terms of I-E based model.

Table 1. Comparison BETWEEN SE Attack Techniques in Terms of I-E Based Human Weakness

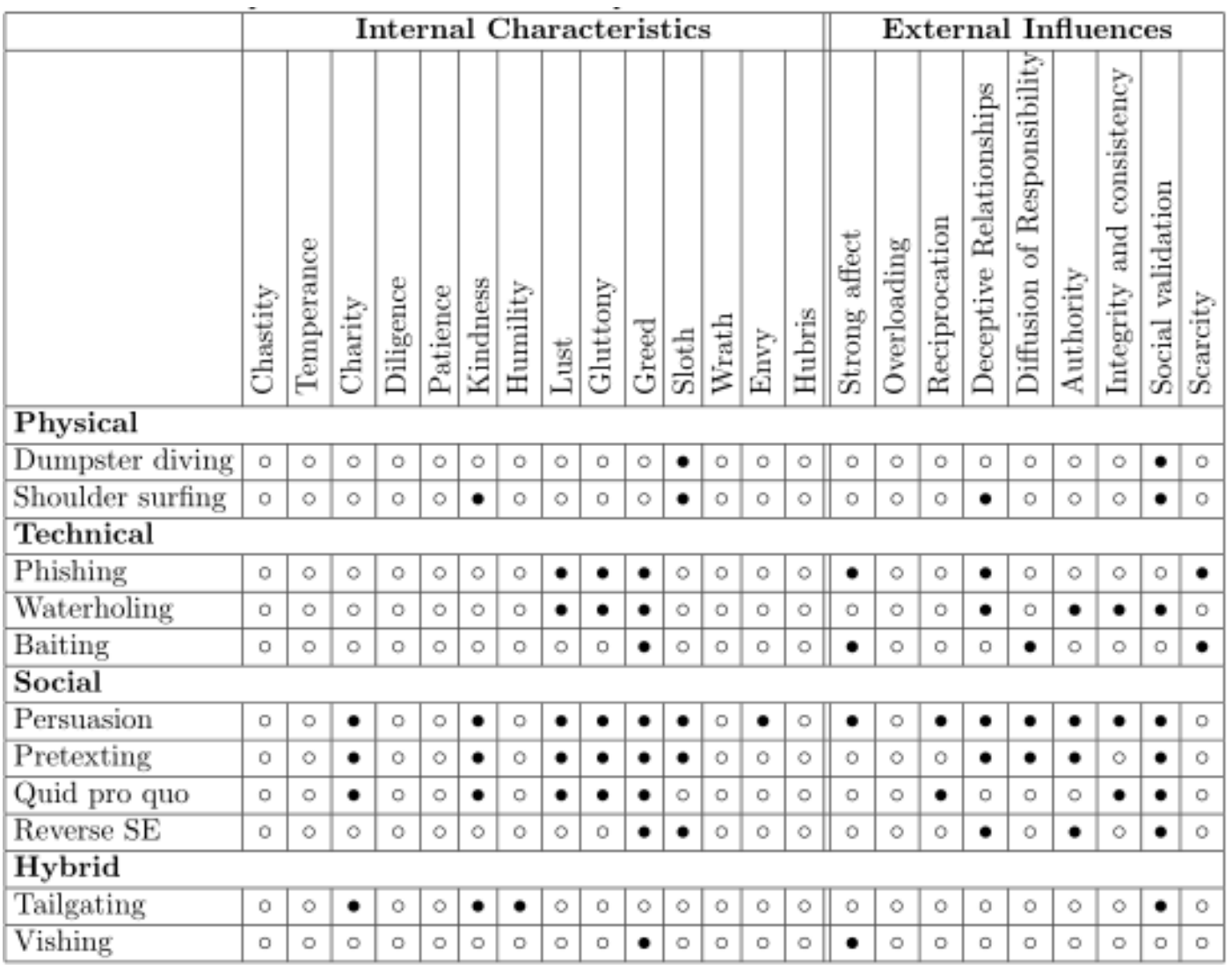

The comparison presents that different attack techniques have different emphasis on exploiting human weaknesses. First, the physical approaches often rely on the human element's incaution under social validations. For example, the company employees often simply drop the unused materials into the trash can, which is a normal social scenario. However, the undestroyed materials leave the opportunity to the adversary to perform the dumpster diving. Also, the regular working scenario will let down one's guard for other colleagues' shoulder surfing. Second, 
to the technical approaches, we find the human element's greed is the main cause of the weakness. It is not strange that the dupe accesses some contents through emails or websites, which raises his interest due to his greed characteristics such as lust and gluttony. For example, some phishing emails or websites often use porn information to lure the dupe to divulge personal information. Third, considering the social approaches, we can discover the fact that both the positive and the negative characteristics can become human weaknesses under certain circumstance influences. The dupe's charity under some social validation can allow the adversary to build trust easily. Also, it is very normal that someone will trust the others who have ever helped him. General employees in a company often comply with the boss's requests and orders as well. Fourth, for the hybrid approaches, on one hand, the tailgating uses the dupe's charity, kindness and humility under some social validation, and for politeness, the dupe performs the action under the adversary's manipulation, which helps the adversary to access the target resource; on the other hand, similar to phishing, the vishing approach is aimed to exploit the dupe's greed to complete the goal.

We discover that the most vulnerable characteristic of human nature is greed that includes lust, gluttony, avarice etc, which can be easily exploited by SE attack techniques under external circumstance influences. It could be attributed to the theory of "selfish-gene" [24] as Dawkins presented. Also, the sloth is a very important vulnerability of human element. Many social engineers actually exploit the poor dupe's lazy personality to complete goals. So, there is an old Chinese saying that "the poor person must have detestable place." In summary, most of human weaknesses are exploited by the technical and the social approaches, and those negative characteristics are much more vulnerable than the positive ones.

\section{DEFENSE MEASURES}

Since threats cannot be eliminated thoroughly but can be reduced by using security measures, in this section, we present some defense measures to fix the human weaknesses to reduce the risk of social engineering attacks. Now that the proposed I-E based model includes two levels, we consider two corresponding categories of defense measures, i.e. subjective and objective, to cope with the weaknesses. The next two subsections describe these two categories of defense measures in detail.

\section{A. Objective Defense Measures}

An objective defense measure is aimed to provide some objective conditions to avoid or reduce the impact of the external circumstance influences over the internal characteristics of human nature.

\section{1) Using standard security policies}

First, well defined and documented security policy is the foundation for defending SE attacks. Using the standard security policy is an effective way to help the organizations train their employees and control security risk. Organizations often use information security management system (ISMS) to provide a framework for information security risk management. ISMS consists of sets of security policies to define, construct, develop and maintain the computer system (including hardware and software resources)-based security within companies. At present, there are several security standards for IT Governance which leads to information security, and the big five of ISMS standards are: ISO/IEC 27001, BS 7799, COBIT, PCI DSS, ITIL \& ISO 2000. These policies dictate the way that the computer resources can be used. However, most security standards and policies are defined to address general information security risks, such as malware, hackers and phishers, which threaten organizations. Hence, these general security policies are ineffective, owing to a failure to acknowledge all that is actually required to cope with SE attacks.

For defending SE attacks, the set of policies provided by the security standards should cover not only the computer-based risks but also the human-based risks. ISO/IEC 27032, extended from ISO/IEC 27001, is a completely new international standard published by ISO that covers the baseline security practices for all stakeholders in cyberspace. In particular, it provides technical guidance for addressing SE attacks. Thus, the organization concerning information security can choose ISO/IEC 27032 to implement the cyber security framework to prevent SE attacks. However, this novel security standard still needs to be validated with respect to how it will turn out in practice and how widely it will be accepted. Using the security standards makes an ease of security measurement.

\section{2) Updating facilities}

If the corporation has a good financial position, it is suggested to update the office facilities. For example, in order to prevent the dumpster diving, the organization should equip the paper shredder to avoid the sensitive information being left in the trash can. Furthermore, using the fingerprinting-based authentication approach to replace the password typing-based access control can help avoid shoulder surfing. Another case in point is to hire security guards at the entrance of building and some restricted areas as a supplement to the electronic access control. All persons entering the building are required to swipe the ID card. The one with no ID entry has to register his information and pass the security check by the security guards.

Besides, it is necessary to apply monitoring facilities to record social activities, which can increase the difficulty of performing social engineering attacks. The phone-call recording is used to capture the social techniques happened in the call center environment, such as the ebank system where the call center agents directly communicate with the social engineers. Indeed, the phone-call recording mechanism has been applied widely in many banks' call centers. Commonly, the user will be notified at the beginning of the conversation by the call 
center agent that this talk will be recorded. Therefore, it can be imagined that even if the social engineer can disguise perfectly and bypass the detection layer, his malicious behavior will have been captured. These data can be used to track the social engineer and even used as the evidence of crime. Also, the digital surveillance is aimed to capture the physical malicious behavior, such as dumpster diving, shoulder surfing and even tailgating. The surveillance camera is a widely used device to facilitate the monitoring task. It can be seen in many public places, e.g. super markets, hospitals, banks etc. Definitely, many enterprises also use the surveillance camera as a security approach. Note that the camera should be not only equipped at the entrance but also fixed inside the enterprises to monitor the potential social engineering behavior from insider. The data captured by the camera can be used to track the social engineer and even applied as the evidence of crime as well.

\section{3) Detecting malicious data}

As stated, the two effective ways to exploit human weaknesses are technical and social techniques. For example, the phishing is often based on the unidirectional communication media while dialog-based attacks are often based on the bidirectional communication media. In order to detect the attack pattern spreading through the digital media, such as email, instant messaging, website etc., it is appropriate to use automated security program. For example, Bhakta and Harris [26] presented a novel approach based on a pre-defined Topic Blacklist (TBL) to detect SE attacks by checking whether the discussion topics of each line of the text generated by the potential attacker match the topics listed in the TBL. The topic blacklist (TBL) is proposed to check if the sender requests sensitive information or not. The TBL is a list of statement topics, which describe a sensitive operation associated to a sensitive data. So, if the request message hits the TBL, the system will make an alert to remind the dupe to raise vigilance.

\section{B. Subjective Defense Measures}

A subjective defense measure is used to improve the human element's subjective wills to overcome the impact of the external circumstance influences on the internal characteristics of human nature.

\section{1) Training human awareness}

Once the foundation of a security policy has been established and approved, all employees should be trained with security awareness. Though the organizations apply appropriate security standards, they still need to train the employees' awareness to defend the SE attacks.

This task can be done by defining the awareness needs of various audience groups within the organization (executives, line managers, users etc.); determining the most effective awareness methods for each audience group (i.e., briefings, messages, courses); developing and disseminating awareness materials (presentations, posters, mailings etc.) regarding the requirement of adherence to the policy. The awareness function also includes the efforts to integrate up-to-date policy compliance and enforcement feedback as well as current threat information, to make the awareness information as topical and realistic as possible. For example, Mataracioglu et al. [27] proposed a qualitative method called security lifecycle model against SE attacks (SLMSEA). Although this approach still mainly focuses on enhancing the individuals' awareness to prevent social engineering, it proposed a comprehensive model consisting of user training, testing, measuring, and result feedback.

However, the conventional human-involved awareness training methods, such as educational courses, routine reminding, interview, awareness quiz and survey etc., are labor intensive, repetitive and even perhaps tedious. At present, there are some automated tools that can be used to train and promote user awareness by simulating real world SE attacks. For example, the King Phisher (https://n0where.net/phishing-campaign-toolkit-kingphisher/) is an open-source tool for automatically training the users' awareness to prevent phishing attacks. It can be used to run campaigns ranging from simple awareness training to more complicated scenarios in which user aware content is served for harvesting credentials.

Nevertheless, the previously mentioned methods are passive solutions that enforce the employees to be aware of the sensitive information protected by the security policy. Indeed, awareness training does not simply require the employees to keep secret of the sensitive information, but desire them to know how to identify confidential information and understand their responsibility to protect it. Thus, a positive method is to combine the employees' profit, which could be the bonus, reward or merit pay, with the sensitive information security. Thereafter, all employees will actively improve their awareness because the information security has been associated to their own financial benefits.

\section{2) Detecting human emotion}

Changes in emotional state have an influence on the individual's cognitive functioning. Hence, the employee's emotional state can affect his awareness of the sensitive information. For example, people often perform abnormal activities under some extreme emotions, such as wrath. However, it is not an easy task to determine one's emotional state, and it is even an impossible task for an individual to adjust his or her own emotional state. This is because individuals have their own perception of emotional state and some individuals are unable to perform this kind of task in a rational way especially when their emotions are irrationally challenged. Besides, some basic concepts of emotional state should be taken into account. First, one's emotional state is something that can stay constant for a long time unless the individual experiences some greatly discomforting incidents, such as economic crisis, health issue, loved ones' death etc., which have an intense effect on his cognitive function. Second, an individual's emotional state can be impacted in a short time when the individual is under attack by the 
SE attacker. Mathews [28] presented that experiencing severe stress would have an influence on the individual's cognitive function. Hence, it is desired to use some emotion detection model for automatically performing this task.

Bezuidenhout et al. [29] proposed an SE attack detection model (SEADM), which can be used by the workers to detect SE attacks from the requesters in a call centre environment. The authors claimed that the social engineers often utilize psychological weaknesses to influence the victim's emotional state and cognitive abilities in order to get objective information. In order to enhance the individual's awareness to the social engineering requests, the paper proposed an automated self-evaluation electronic questionnaire. If the individual is detected too emotional, the call or the email request will be transferred to another individual. However, this strategy could initiate the work responsibility shift and even promote further frustration within all the individuals involved. The detection of one's emotional state by the first SEADM is subjective, and it is impossible to make instantaneous decision whilst working under pressure. Thus, Mouton et al. [30] improved the SEADM by proposing and incorporating a cognitive functioning psychological measure to determine the emotional state and decision-making ability. Nevertheless, the two previous papers related to SEADM only focus on the call centre environment. Mouton et al. [31] therefore proposed a revised version of SE attack detection model, namely SEADMv2, extending the model to much more different SE scenarios.

\section{CONCLUSIONS}

Social engineering attack is an open and big challenge in the area of cyber crime in the modern human-centered environment. In this paper, we revisited the overview of social engineering attack and identified the root problem, i.e. the human weakness. We captured two essential levels - internal characteristics of human nature and external circumstance influences - that shape the human weakness for social engineering. Therefore, we proposed a novel I-E based model of human weakness and defined these two levels' terminologies. We classified the characteristics into two categories - positive and negative, and cited the "seven virtues and sins" in the Catholic catechism to make up the cardinal characteristics. Also, we presented nine common circumstance influences. Using this new I-E based model we analyzed a number of typical social engineering attack techniques. We discovered that the human negative characteristics, such as greed and sloth, are much more vulnerable than those positive ones, which can all be exploited by SE techniques. Finally, we presented a number of defense measures to fix the human weaknesses. These defense measures are categorized into objective approaches and subjective approaches according to the I-E based model. In summary, this work provides a new perspective to investigate social engineering, and we hope that it can help the related security researchers get insights into the social engineering and enhance the future research.

\section{REFERENCES}

[1] Hossein Bidgoli. Handbook of Information Security, Information Warfare, Social, Legal, and International Issues and Security Foundations (Handbook of Information Security). John Wiley \& Sons, Inc., New York, NY, USA, 2006.

[2] Ji-Xuan Feng and Janet Hughes. Analyzing privacy and security issues in the information age - an ethical perspective. WSEAS Trans. Info. Sci. and App., 6(1):126-135, January 2009.

[3] RC Joshi and Anjali Sardana. Honeypots: a new paradigm to information security. CRC Press, 2011.

[4] Kevin D Mitnick and William L Simon. The art of deception: Controlling the human element of security. John Wiley \& Sons, 2011.

[5] Verizon RISK Team. 2015 data breach investigations report. 2015.

[6] Ponemon Institute. The cost of phishing and value of employee training, Aug 2015.

[7] Symantec Enterprise. Internet security threat report 2015, 2015.

[8] Christopher Hadnagy. Social engineering: The art of human hacking. John Wiley \& Sons, 2010.

[9] Ian Mann. Hacking the human: social engineering techniques and security countermeasures. Gower Publishing, Ltd., 2012.

[10] A. Avizienis, J. C. Laprie, B. Randell, and C. Landwehr. Basic concepts and taxonomy of dependable and secure computing. IEEE Transactions on Dependable and Secure Computing, 1(1):11-33, Jan 2004.

[11] Simon Hansman and Ray Hunt. A taxonomy of network and computer attacks. Computers \& Security, 24(1):31 $-43,2005$.

[12] Chris Simmons, Charles Ellis, Sajjan Shiva, Dipankar Dasgupta, and Qishi Wu. Avoidit: a cyber attack taxonomy. Technical Report CS-09-003, University of Memphis, Aug 2009.

[13] RP Van Heerden, Barry Irwin, ID Burke, and L Leenen. A computer network attack taxonomy and ontology. International Journal of Cyber Warfare and Terrorism (IJCWT), 2(3):12-25, 2012.

[14] Katharina Krombholz, Heidelinde Hobel, Markus Huber, and Edgar Weippl. Advanced social engineering attacks. Journal of Information Security and Applications, 22(C):113-122, June 2015.

[15] Ryan Heartfield and George Loukas. A taxonomy of attacks and a survey of defence mechanisms for semantic social engineering attacks. ACM Comput. Surv., 48(3):37:1-37:39, December 2015.

[16] CERT-UK. Common cyber attacks: Reducing the impact, 2015.

[17] Francois Mouton, Louise Leenen, and H.S. Venter. Social engineering attack examples, templates and scenarios. Computers \& Security, 59:186 - 209, 2016.

[18] Marcus Nohlberg and Stewart Kowalski. The cycle of deception: a model of social engineering attacks, defenses and victims. In Second International Symposium on Human Aspects of Information Security and Assurance (HAISA 2008), pages 1-11, Plymouth, UK, July 8-9 2008.

[19] F. Mouton, M. M. Malan, L. Leenen, and H. S. Venter. Social engineering attack framework. In 2014 Information Security for South Africa, pages 1-9, Aug 2014. 
[20] Francois Mouton, Louise Leenen, Mercia M. Malan, and H. S. Venter. Towards an ontological model defining the social engineering domain. In 11th IFIP TC 9 International Conference on Human Choice and Computers (HCC11 2014), pages 266-279, Turku, Finland, July 31 - August 1, 2014.

[21] Jose J. Gonzalez, Jose M. Sarriegi, and Alazne Gurrutxaga. A framework for conceptualizing social engineering attacks, pages 79-90. Samos, Greece, August 31 - September 1, 2006.

[22] Pekka Tetri and Jukka Vuorinen. Dissecting social engineering. Behaviour \& Information Technology, 32(10):1014-1023, 2013.

[23] Sherly Abraham and InduShobha Chengalur-Smith. An overview of social engineering malware: trends, tactics, and implications. Technology in Society, 32(3):183 196, 2010.

[24] Richard Dawkins. The selfish gene. 1976.

[25] Dale Carnegie. How to win friends and influence people. Simon and Schuster, 2010.

[26] R. Bhakta and I. G. Harris. Semantic analysis of dialogs to detect social engineering attacks. In Semantic Computing (ICSC), 2015 IEEE International Conference on, pages 424-427, Feb 2015.

[27] Tolga Mataracioglu, SevgiÖzkan, and Ray Hackney. Towards a security lifecycle model against social engineering attacks: SLM-SEA. CoRR, abs/1507.02458, 2015.

[28] Andrew Mathews. Why worry? the cognitive function of anxiety. Behaviour Research and Therapy, 28(6):455 $-468,1990$.

[29] M. Bezuidenhout, F. Mouton, and H. S. Venter. Social engineering attack detection model: SEADM. In 2010 Information Security for South Africa, pages 1-8, Aug 2010.

[30] Francois Mouton, Mercia M Malan, and Hein S Venter. Development of cognitive functioning psychological measures for the seadm. In HAISA, pages 40-51, 2012.

[31] F. Mouton, L. Leenen, and H. S. Venter. Social engineering attack detection model: Seadmv2. In 2015 International Conference on Cyberworlds $(\mathrm{CW})$, pages 216-223, Oct. 2015.

\section{Authors' Profiles}

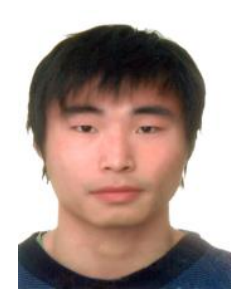

Wenjun Fan is currently a Phd candidate in the Department of Telematics Engineering (DIT) at Technical University of Madrid (UPM) since September 2011. His research areas include network \& system security, information security, intrusion detection and response.

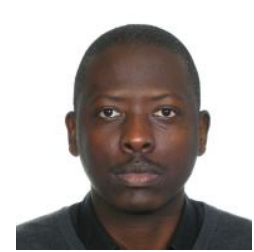

Kevin Lwakatare received his Bachelor of engineering in Information technology from Helsinki Metropolitan University of Applied Science (HMUAS) in 2011 in Finland, After working for two years as software developer, he obtained a scholarship to study in Estonia and then received a Master of Science degree in cyber security from Tallinn university of technology in 2016. Currently he works as cyber security analyst and independent cyber security researcher.

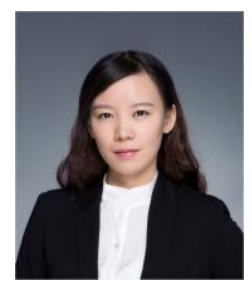

Rong Rong is a alumna of IE Business School in Madrid, Spain, where she got the master degree of International Business Management in 2013. She also has a master of philosophy degree in the Chinese University of Hong Kong with the major in Medical Sciences. With a great interest in social psychology, she now works actively in the venture capital investment of Chinese medical industry and social welfare business.

How to cite this paper: Wenjun Fan, Kevin Lwakatare, Rong Rong,"Social Engineering: I-E based Model of Human Weakness for Attack and Defense Investigations", International Journal of Computer Network and Information Security(IJCNIS), Vol.9, No.1, pp.1-11, 2017.DOI: 10.5815/ijcnis.2017.01.01 